Acta Crystallographica Section E

Structure Reports

Online

ISSN 1600-5368

\title{
Zwitterionic 2-(methylamino)ethanesulfonic acid
}

The title compound, $N$-methyltaurine, $\mathrm{C}_{3} \mathrm{H}_{9} \mathrm{NO}_{3} \mathrm{~S}$, was isolated from the marine sponge Xestospongia pacifica from Swain Reefs, Queensland. The crystal structure displays extensive $\mathrm{N}-\mathrm{H}$. . O hydrogen bonding interactions between the amine $\mathrm{H}$ atoms and sulfonyl $\mathrm{O}$ atoms in the zwitterionic molecule.

\section{Comment}

In a recent study, $N$-methyltaurine is reported to be a major osmolyte in a specimen of the tubeworm Lamellibrachia sp. (Yin et al., 2000). Osmolytes are small organic molecules that regulate cell volume by countering osmotic pressure exerted by sea water and it seems reasonable to assume that $N$-methyltaurine performs a similar role in Xestospongia pacifica.

Molecules of 2-(methylamino)ethanesulfonic acid, (I), crystallize in the zwitterionic form with the sulfonic acid $\mathrm{H}$ atom transferred to the $\mathrm{N}$ atom (Fig. 1 and Table 1 ). The bond lengths and angles are in accord with conventional values (Allen et al., 1987) and related structures (Görbitz et al., 2000).

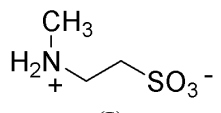

(I)

In the crystal structure, the molecules are linked via a number of $\mathrm{N}-\mathrm{H} \cdots \mathrm{O}$ hydrogen bonds to form a threedimensional network (Table 2).

\section{Experimental}

Compound (I) was isolated from a methanol extract of the marine sponge Xestospongia pacifica from Swain Reefs, Queensland. The extract was subjected to repeated gel-permeation chromatography on Sephadex LH-20 in methanol. Crystals of (I) were obtained on slow evaporation of the methanol from the parent fraction; m.p. $520 \mathrm{~K}$ (with decomposition). $\delta_{H}$ (400 MHz, DMSO- $d_{6}$, p.p.m.): 4.1 (2H, brs, $\left.\mathrm{NH}_{2}\right), 3.16\left(3 \mathrm{H}, s, \mathrm{~N}-\mathrm{CH}_{3}\right), 3.14\left(2 \mathrm{H}, t, J_{2,1}=6.4 \mathrm{~Hz}, \mathrm{H} 2\right), 2.77(2 \mathrm{H}, t$, $\left.J_{1,2}=6.4 \mathrm{~Hz}, \mathrm{H} 1\right),($ ESMS +$): 161.8\left(\mathrm{MNa}^{+}\right),($ESMS -$) 137.8(M-\mathrm{H})$.

\section{Crystal data}

$\mathrm{C}_{3} \mathrm{H}_{9} \mathrm{NO}_{3} \mathrm{~S}$

$M_{r}=139.18$

Orthorhombic, $P 22_{1} 22_{1}$

$a=9.061(3) \AA$

$b=11.931(3) \AA$

$c=5.4924(15) \AA$

$V=593.8(3) \AA^{3}$

$Z=4$

$D_{x}=1.557 \mathrm{Mg} \mathrm{m}^{-3}$
Received 14 April 2003 Accepted 22 April 2003 Online 30 April 2003 
Data collection

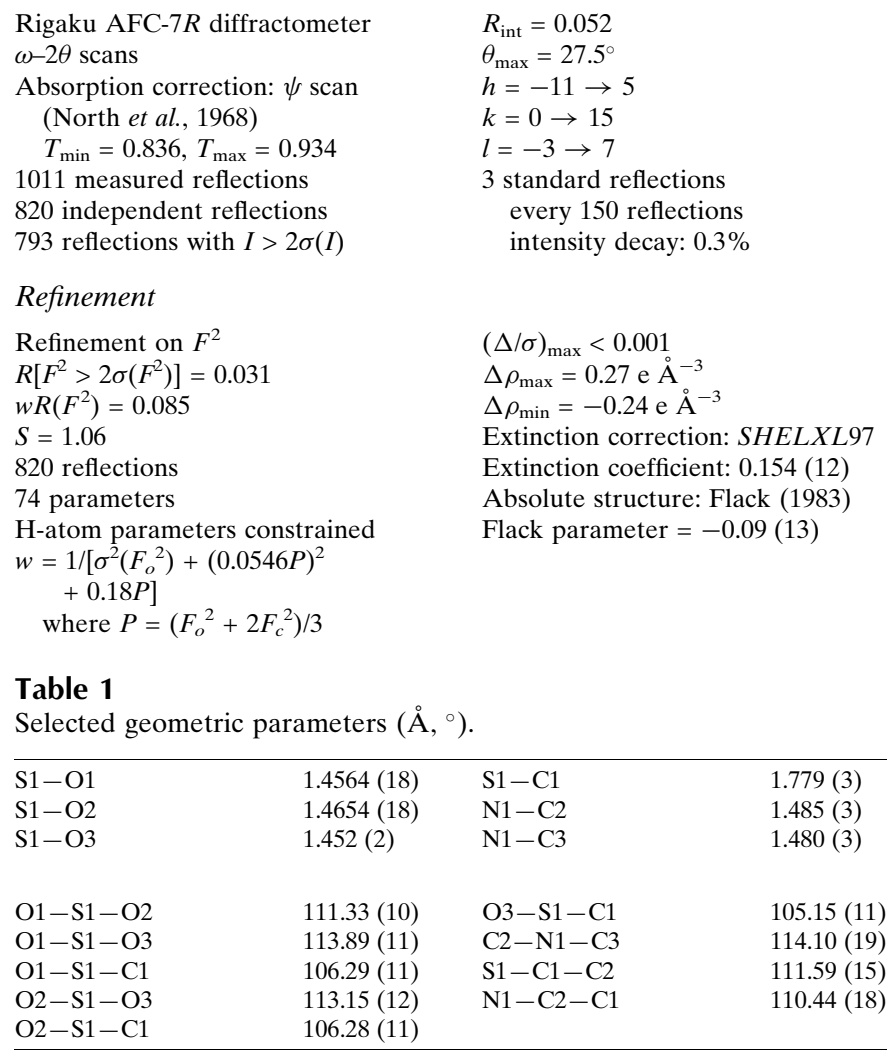

\section{Table 2}

Hydrogen-bonding geometry $\left(\AA{ }^{\circ}\right)$.

\begin{tabular}{lllll}
\hline$D-\mathrm{H} \cdots A$ & $D-\mathrm{H}$ & $\mathrm{H} \cdots A$ & $D \cdots A$ & $D-\mathrm{H} \cdots A$ \\
\hline $\mathrm{N} 1-\mathrm{H} 5 \cdots \mathrm{O} 1^{\mathrm{i}}$ & 0.85 & 2.13 & $2.862(3)$ & 144 \\
$\mathrm{~N} 1-\mathrm{H} 5 \cdots 3^{\mathrm{ii}}$ & 0.85 & 2.52 & $2.945(3)$ & 112 \\
$\mathrm{~N} 1-\mathrm{H} 6 \cdots \mathrm{O} 2^{\mathrm{iii}}$ & 0.85 & 2.02 & $2.798(3)$ & 152 \\
\hline
\end{tabular}

Symmetry codes: (i) $\frac{1}{2}-x,-y, \frac{1}{2}+z$; (ii) $x-\frac{1}{2}, \frac{1}{2}-y, 2-z$; (iii) $x-\frac{1}{2}, \frac{1}{2}-y, 1-z$.

$\mathrm{H}$ atoms were constrained as riding atoms, with $\mathrm{C}-\mathrm{H}$ distances of $0.95 \AA$ and $\mathrm{N}-\mathrm{H}$ distances of $0.85 \AA$. $U_{\text {iso }}(\mathrm{H})$ values were set to $1.2 U_{\text {eq }}$ for the parent atom.

Data collection: MSC/AFC-7 Diffractometer Control Software (Molecular Structure Corporation, 1999); cell refinement: $M S C / A F C$ -

7 Diffractometer Control Software; data reduction: TEXSAN

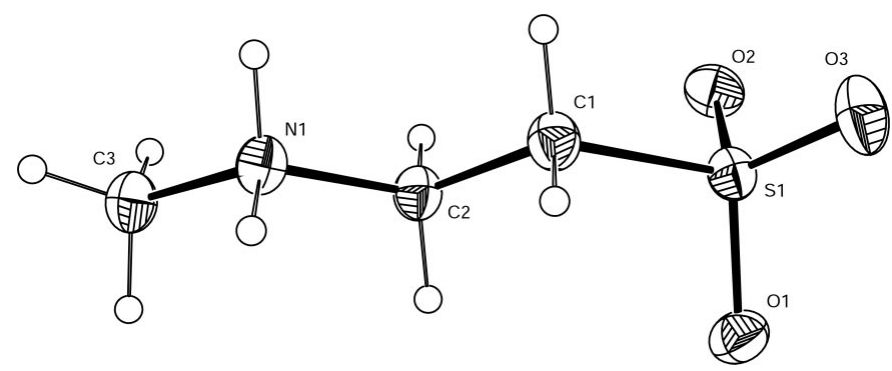

Figure 1

ORTEP-3 (Farrugia, 1997) plot, showing the atomic numbering scheme for the molecule of (I). Displacement ellipsoids for non-H atoms are drawn at the $30 \%$ probability level.

(Molecular Structure Corporation, 1997-2001); program(s) used to solve structure: TEXSAN; program(s) used to refine structure: TEXSAN and SHELXL97 (Sheldrick, 1997); molecular graphics: PLATON (Spek, 1980-2001) and ORTEP-3 (Farrugia, 1997); software used to prepare material for publication: TEXSAN and PLATON.

The marine sponge Xestospongia pacifica Kelly-Borges \& Bergquist, 1998 (phylum Porifea, class Demospongiae, order Haplosclerida, family Petrosiidae) was collected by hand using SCUBA from Swain Reefs, Queensland, Australia, at a depth of $28 \mathrm{~m}$ by Dr John Hooper and co-workers from the Sessile Marine Invertebrate section, Queensland Museum. A voucher sample (G305705) is lodged at the Queensland Museum, Brisbane, Australia.

\section{References}

Allen, F. H., Kennard, O., Watson, D. G., Brammer, L., Orpen, A. G. \& Taylor, R. (1987). J. Chem. Soc. Perkin Trans. 2, pp. S1-19.

Farrugia, L. J. (1997). J. Appl. Cryst. 30, 565.

Flack, H. D. (1983). Acta Cryst. A39, 876-881.

Görbitz, C. H., Prydz, K. \& Ugland, S. (2000). Acta Cryst. C56, e23-e24.

Molecular Structure Corporation (1999). MSC/AFC-7 Diffractometer Control Software. Windows Version 1.02. MSC, 9009 New Trails Drive, The Woodlands, TX 77381, USA.

Molecular Structure Corporation (1997-2001). TEXSAN for Windows. Version 1.06. MSC, 9009 New Trails Drive, The Woodlands, TX 77381, USA. North, A. C. T., Phillips, D. C. \& Mathews, F. S. (1968). Acta Cryst. A24, 351359.

Sheldrick, G. M. (1997). SHELXL97. University of Göttingen, Germany. Spek, A. L. (1980-2001). PLATON for Windows. Version 121201. University of Utrecht, The Netherlands.

Yin, M., Palmer, H. R., Fyfe-Johnson, A. L., Bedford, J. J., Smith, R. A. J. \& Yancey, P. H. (2000). Physiol. Biochem. Zool. 73, 629-637. 